\title{
SUMMABILITY AND ANALYTIC CONTINUATION
}

\author{
V. F. COWLING ${ }^{1}$
}

1. Introduction. In this paper we describe a new family of Toeplitz summability methods, and we study the regions in which these methods sum a Taylor series to the analytic continuation of the function which it represents.

Let $A=\left(a_{n m}\right)$ and $x=\left\{s_{m}\right\}(n, m=0,1, \cdots)$ be a matrix and a sequence of complex numbers, respectively. We write formally

$$
t_{n} \equiv A_{n}(x) \equiv \sum_{m=0}^{\infty} a_{n m} s_{m}
$$

and say that the sequence $x$ (and the corresponding series $\sum_{m=0}^{\infty}\left(s_{m}-s_{m-1}\right)$, with $\left.s_{-1}=0\right)$ is summable $A$ to the sum $t$ if each of the series in (1) converges and $\lim t_{n}$ exists and equals $t$. We say that the method $A$ is regular provided it sums every convergent sequence to its limit. The method $A$ is regular if and only if

$$
\begin{aligned}
\sum_{m=0}^{\infty}\left|a_{n m}\right| & \leqq K & (n=0,1, \cdots), \\
\lim _{n \rightarrow \infty} a_{n m} & =0 & (m=0,1, \cdots), \\
\lim _{n \rightarrow \infty} \sum_{m=0}^{\infty} a_{n m} & =1, &
\end{aligned}
$$

where $K$ is a constant independent of $n$ (cf. Toeplitz ${ }^{2}$ [2]).

2. The methods $F^{r}$. For each constant $r(r \neq 1)$ the element of the matrix $A=F^{r}$ shall be defined by the equations

$$
\begin{aligned}
& a_{n m}(r)=0 \\
& (m<n), \\
& a_{n m}(r)=(1-r)^{n+1} C_{m, n} r^{m-n} \\
& (m \geqq n) \text {. }
\end{aligned}
$$

We note first that, for $n=0,1, \cdots$ and $|r|<1$,

$$
\sum_{m=0}^{\infty} a_{n m}(r)=(1-r)^{n+1} \sum_{m=n}^{\infty} C_{m, n} r^{m-n}=1
$$

Presented to the Society, December 30, 1948; received by the editors June 2, 1949.

1 The author wishes to acknowledge the constructive criticism of Professor G. Piranian on this paper.

2 Numbers in brackets refer to the bibliography at the end of the paper. 
and

$$
\sum_{m=0}^{\infty}\left|a_{n m}(r)\right|=\left(\frac{1-r}{1-|r|}\right)^{n+1}
$$

If on the other hand $|r| \geqq 1 \quad(r \neq 1)$, then $\sum_{m=0}^{\infty}\left|a_{n m}(r)\right|=\infty$ $(n=0,1, \cdots)$. Therefore the method $F^{r}$ is regular if and only if $r$ is real and satisfies the condition $0 \leqq r<1$.

THEOREM $^{3}$ 2.1. If $A B$ denotes the matrix product of $A$ and $B$, $F^{r_{1}} F^{r_{2}}=F^{r_{2}} F^{r_{1}}=F^{r}$, where $r=r_{1}+r_{2}-r_{1} r_{2}$.

Let $F^{r_{1}}=\left(a_{n m}\right), F^{r_{2}}=\left(b_{n m}\right), F^{r_{1}} F^{r_{2}}=\left(c_{n m}\right)$ and $F^{r_{2}} F^{r_{1}}=\left(\gamma_{n m}\right)$. Then $c_{n m}=0$ when $n>m$. When $n \leqq m$,

$$
\begin{aligned}
c_{n m} & =\sum_{k=0}^{\infty} a_{n k} b_{k m} \\
& =\left(1-r_{1}\right)^{n+1} r_{1}^{-n} r_{2}^{m} \sum_{k=n}^{m} r_{1}^{k} r_{2}^{-k}\left(1-r_{2}\right)^{k+1} C_{k, m} C_{n, k} \\
& =\left(1-r_{1}\right)^{n+1} r_{1}^{-n} r_{2}^{m}\left(\frac{r_{1}}{r_{2}}\right)^{n}\left(1-r_{2}\right)^{n+1} C_{m, n} \sum_{k=0}^{m-n} C_{m-n, k}\left(\frac{r_{1}}{r_{2}}-r_{1}\right)^{k} \\
& =\left(1-r_{1}\right)^{n+1}\left(1-r_{2}\right)^{n+1} C_{m, n}\left(r_{1}+r_{2}-r_{1} r_{2}\right)^{m-n} \\
& =\left[1-\left(r_{1}+r_{2}-r_{1} r_{2}\right)\right]^{n+1} C_{m, n}\left(r_{1}+r_{2}-r_{1} r_{2}\right)^{m-n} .
\end{aligned}
$$

Since the last expression is symmetric in $r_{1}$ and $r_{2}, \gamma_{n m}=c_{n m}$, the proof is complete. We note the corollary that, for $r \neq 1$, the matrix $F^{r}$ has the inverse $F^{p}$, where $\rho=-r /(1-r)$.

Theorem 2.2. If $r_{1}$ and $r_{2}$ are real constants $\left(0 \leqq r_{1}<r_{2}<1\right)$, every bounded sequence summed by $F^{r_{1}}$ is summed by $F^{r_{2}}$, and to the same sum.

Let $r=\left(r_{2}-r_{1}\right) /\left(1-r_{1}\right)$; then the method $F^{r}$ is regular, and $F^{r 2}$ $=F^{r} F^{r}$, by Theorem 2.1. The present theorem now follows from a remark by Agnew [1] (cf. p. 328).

3. Summability of Taylor series. Henceforth, $\left\{u_{m}\right\}$ and $\left\{s_{m}\right\}$ shall denote sequences of complex numbers related by the equations

$$
s_{m}=u_{0}+u_{1}+\cdots+u_{m} \quad(m=0,1, \cdots) .
$$

Lemma. If

${ }^{3}$ The author is indebted to A. Wilansky for helpful discussion regarding this result. 
(i) the series $\sum u_{m} z^{m}$ has a positive radius of convergence $R$;

(ii) $|r|<R$;

(iii) $t_{n}=(1-r)^{n} \sum_{m=n}^{\infty} C_{m, n} U_{m} r^{m-n}(n=0,1, \cdots)$;

(iv) $T_{n}=t_{0}+t_{1}+\cdots+t_{n}(n=0,1, \cdots)$; then

$$
T_{n}=(1-r)^{n+1} \sum_{m=n}^{\infty} C_{m, n} s_{m} r^{m-n} \quad(n=0,1, \cdots) .
$$

Equation (6) asserts that a certain sum of $n$ Taylor series is equal to another Taylor series. All the series in question converge because, for any fixed positive integer $n$, the respective radii of convergence of the four series

$$
\sum_{m=0}^{\infty} u_{m} z^{m}, \quad \sum_{m=0}^{\infty} s_{m} z^{m}, \quad \sum_{m=0}^{\infty} C_{m, n} u_{m} z^{m}, \quad \sum_{m=0}^{\infty} C_{m, n} s_{m} z^{m}
$$

are equal. The validity of the equation will now be established by induction.

For $n=0$, the equation (6) reduces to the identity

$$
(1-r) \sum_{m=0}^{\infty} s_{m} r^{m}=\sum_{m=0}^{\infty} u_{m} r^{m}
$$

But if the equation holds for $n=k$, that is, if

$$
T_{k}=\frac{(1-r)^{k+1}}{r^{k}} \sum_{m=k}^{\infty} C_{m, k} S_{m} r^{m},
$$

then

$$
\begin{aligned}
T_{k+1}= & T_{k}+t_{k+1} \\
= & \left(\frac{1-r}{r}\right)^{k+1}\left\{\sum_{m=k}^{\infty} C_{m, k} s_{m} r^{m+1}+\sum_{m=k+1}^{\infty} C_{m, k+1}\left(s_{m}-s_{m-1}\right) r^{m}\right\} \\
= & \left(\frac{1-r}{r}\right)^{k+1}\left\{\sum_{m=k+1}^{\infty}\left[C_{m, k} r+C_{m, k+1}-C_{m+1, k+1} r\right] s_{m} r^{m}\right. \\
& \left.+\left[C_{k, k}-C_{k+1, k+1}\right] s_{k} r^{k+1}\right\} \\
= & \left(\frac{1-r}{r}\right)^{k+1} \sum_{m=k+1}^{\infty}\left\{C_{m, k+1}-\left[C_{m+1, k+1}-C_{m, k}\right] r\right\} s_{m} r^{m} \\
= & \frac{(1-r)^{k+2}}{r^{k+1}} \sum_{m=k+1}^{\infty} C_{m, k+1} s_{m} r^{m} .
\end{aligned}
$$


In other words, equation (6) also holds for $n-k+1$. This proves the lemma. From the lemma it follows that if $0<|r|<R$, where $R$ is the radius of convergence of the series $\sum u_{m} z^{m}$, the relation

$$
\sum_{n=0}^{\infty}\left(\frac{1-r}{r}\right)^{n} \sum_{m=n}^{\infty} C_{m, n} u_{m} r^{m}=\lim _{n \rightarrow \infty} \frac{(1-r)^{n+1}}{r^{n}} \sum_{m=n}^{\infty} C_{m, n} s_{m} r^{m}
$$

holds in the sense that the existence of either member implies the existence, with the same value, of the other member. The following result is now immediate:

THEOREM 3.1. If the series $\sum_{m=0}^{\infty} u_{m} z^{m}$ has a positive radius of convergence $R$, the series $\sum u_{m}$ is summable $F^{r}$ to the sum $L$ for any constant $r(0<|r|<R)$ for which the series

$$
\sum_{m=0}^{\infty}\left(\frac{1-r}{r}\right)^{n} \sum_{m=n}^{\infty} C_{m, n} u_{m} r^{m}
$$

converges to $L$.

4. Analytic continuation by means of the methods $F^{r}$. Let $D$ be a simply connected region in the complex plane, $C$ a simple closed Jordan curve lying in $D$ and bounding the finite region $D^{\prime}$. Corresponding to each complex number $r(r \neq 1)$ we define an open set $R(r, C)$ as follows: $R(r, C)$ is the set of all points in $D^{\prime}$ for which $|z-r z|<|t-r z|$ whenever $t$ lies on $C$. A set $B$ shall be said to be of type $R^{*}(r, D)$ if $B$ is closed and if $C$ can be chosen in such a way that $B$ is a subset of $R(r, C)$. In the following theorem, $f^{(n)}(r z)$ denotes the $n$th derivative with respect to $w$ of $f(w)$ at the point $w=r z$.

THEOREM 4.1. If $f(z)$ is uniform and regular in the region $D, r$ is a complex constant $(r \neq 1)$, and $B$ is a set of type $R^{*}(r, D)$, then the series

$$
\sum_{n=0}^{\infty} f^{(n)}(r z)(1-r)^{n} z^{n} / n !
$$

converges to $f(z)$ uniformly in $B$.

If $z$ lies in $B$,

$$
\begin{aligned}
f(z) & =\frac{1}{2 \pi i} \int_{C} \frac{f(t) d t}{t-z}=\frac{1}{2 \pi i} \int_{C} \frac{f(t)}{t-r z} \frac{1}{1-(z-r z) /(t-r z)} d t \\
& =\frac{1}{2 \pi i} \int_{C} \frac{f(t)}{t-r z} \sum_{n=0}^{\infty}\left(\frac{z-r z}{t-r z}\right)^{n} d t,
\end{aligned}
$$

where $C$ is a curve in $D$ such that $B$ is a subset of $R(r, C)$. The series 
in the last integrand converges uniformly with respect to $t$ on $C$ and $z$ in $B$, because, for such values of $t$ and $z$,

$$
|(z-r z) /(t-r z)|<K<1 \text {, }
$$

where the constant $K$ depends only on $B$ and $C$. Therefore

$$
f(z)=\sum_{n=0}^{\infty}(z-r z)^{n} \frac{1}{2 \pi i} \int_{C} \frac{f(t) d t}{(t-r z)^{n+1}} .
$$

But the point $r z$ is inside of the curve $C$; for otherwise the line segment joining the points $z$ and $r z$ would meet the curve $C$ at some point $t^{\prime}$, the inequality $\left|(z-r z) /\left(t^{\prime}-r z\right)\right|<1$ would not be satisfied, and the point $z$ would not lie in $B$. Therefore the last integral has the value $2 \pi i f^{(n)}(r z) / n^{\prime}$, and the theorem is proved.

The hypothesis that the region $D$ is simply connected will now be removed. Let $D$ be a connected open set, $E$ its boundary, and $r$ a complex constant $(r \neq 1)$. By $Q(r, D)$ we shall denote the set of all points in $D$ for which the inequality $|z-r z|<|t-r z|$ is satisfied whenever $t$ lies in $E$.

THEOREM 4.2. Let the function $f(z)$ be regular and uniform in the bounded region $D$, and let $r$ be a complex constant $(r \neq 1)$. Then the series (7) converges to $f(z)$ absolutely in $Q(r, D)$, and the absolute convergence is uniform in every closed subset of $Q(r, D)$.

Let $B$ be a closed subset of $Q(r, D)$, and $z_{0}$ a point in $B$. It is to be shown that the series

$$
\sum_{n=0}^{\infty} f^{(n)}\left(r z_{0}\right)(1-r)^{n} z_{0}^{n} / n !
$$

converges to $f\left(z_{0}\right)$, and that the absolute convergence of the series (9) is uniform with respect to $z_{0}$ in $B$.

We observe that (8) is the Taylor series of $f\left(z_{0}\right)$ about the point $z=r z_{0}{ }^{4}$ Since $B$ is a closed subset of $Q(r, D)$, there exists a positive number $\epsilon$ such that $\left|\left(z_{0}-r z_{0}\right) /\left(t-r z_{0}\right)\right|<1-2 \epsilon$ when $z_{0}$ is in $B$ and $t$ is on $E$. The Cauchy estimate for the coefficients of our Taylor series gives the result

$$
\left|f^{(n)}\left(r z_{0}\right) / n !\right|<M\left(r z_{0}\right) /\left[(1-\epsilon) \delta_{0}\right]^{n},
$$

where $\delta_{0}$ is the distance from $r z_{0}$ to the set $E$ and $M\left(r z_{0}\right)$ denotes the maximum modulus of $f(z)$ on the circle $\left|z-r z_{0}\right|=(1-\epsilon) \delta_{0}$. As the

4 The author is indebted to A. M. Gleason who pointed out this fact in an oral communication. 
point $z_{0}$ ranges over the set $B$, the points

$$
z=r z_{0}+e^{i \theta}(1-\epsilon) \delta_{0} \quad(0 \leqq \theta<2 \pi)
$$

range over a subset of $D$ which is bounded away from the set $E$, and therefore the quantities $M\left(r z_{0}\right)$ have a common finite upper bound $M$. It follows that

$$
\left|f^{(n)}\left(r z_{0}\right)(1-r)^{n} \frac{z^{n}}{n !}\right|<\frac{\left[(1-2 \epsilon) \delta_{0}\right]^{n} M}{\left[(1-\epsilon) \delta_{0}\right]^{n}}<(1-\epsilon)^{n} M,
$$

and the theorem is proved.

THEOREM 4.3. Let $D$ be a connected open set containing the origin, $f(z)$ a uniform function regular in $D$; let the series $\sum a_{m} z^{m}$ converge to $f(z)$ in a circle of radius $\rho$; and let $r$ be a complex constant $(r \neq 1)$. Then, in every closed set which is contained in the intersection of the set $Q(r, D)$ and the circle $|z|<\rho /|r|$, the series $\sum a_{m} z^{m}$ is uniformly absolutely summable $F^{r}$ to $f(z)$.

If $r=0$, the set $Q(r, D)$ is contained in the circle $|z|<\rho$, and the result is trivial. To prove the theorem for $r \neq 0$, we observe first that the series

$$
\sum_{m=n}^{\infty} a_{m} m(m-1) \cdots(m-n+1)(r z)^{m-n}
$$

converges to $f^{(n)}(r z)$ in the region $|z|<\rho /|r|$. It follows that the series (7) can be written in the form

$$
\sum_{n=0}^{\infty} \sum_{m=n}^{\infty} a_{m} C_{m, n}(1-r)^{n} z^{m} r^{m-n}=\sum_{n=0}^{\infty}\left(\frac{1-r}{r}\right)^{n} \sum_{m=n}^{\infty} C_{m, n} r^{m} a_{m} z^{m},
$$

and upon application of Theorem 3.1 (with $u_{m}=a_{m} z^{m}$ ) the present theorem becomes a corollary of Theorem 4.2. We note that we have established analytic continuation of the function represented by the series $\sum a_{m} z^{m}$ by means of summability methods which need not even be regular.

5. Summability of a special series. We now subject the methods $F^{r}$ to the customary test of applying them to the series $\sum z^{n}$. Here, the sole singularity of the function $f(z)$ is the point $z=1$, and the boundary of the region $D$ can therefore be taken to consist of the point $z=1$ together with the circle $|z|=K$, where $K$ is arbitrarily large. We shall restrict our considerations to the case where $F^{r}$ is regular, that is, where $r$ is real and $0 \leqq r<1$. If $z$ is any point in the plane, the 
inequality $|z-r z|<|t-r z|$ is certainly satisfied if $t$ is sufficiently large. It remains to examine the inequality for the case where $t=1$. Here we have, with $z=x+i y$,

$$
\begin{aligned}
\left(x^{2}+y^{2}\right)(1-r)^{2} & <(1-r x)^{2}+r^{2} y^{2}, \\
(1-2 r)\left(x^{2}+y^{2}\right) & <1-2 r x .
\end{aligned}
$$

Theorem 4.3 now gives the following result: If $0<r<1 / 2, F^{r}$ sums the series $\sum z^{m}$ to the function $1 /(1-z)$ in the intersection of the region $|z|<1 / r$ with the interior of the circle having its center at $z$ $=-r /(1-2 r)$ and passing through the point $z=1$. If $1 / 2<r<1, F^{r}$ sums the series in the intersection of the region $|z|<1 / r$ with the exterior of the circle having its center at $z=r /(2 r-1)$ and passing through the point $z=1$. The method $F^{1 / 2}$ sums the series in the intersection of the region $|z|<2$ with the half-plane $x<1$.

Finally, we recall that in $\S 2$ the relation $F^{r_{2}} \supset F^{r_{1}}\left(0 \leqq r_{1}<r_{2}<1\right)$ was shown to hold in the space of bounded sequences. The relation does not hold in the space of all sequences. For we have now established that the series $\sum(-5 / 3)^{n}$ is summable $F^{1 / 2}$; on the other hand, the transform of this series by $F^{r}$ does not even exist when $r \geqq 3 / 5$.

Added in proof: After the present paper had been accepted for publication, an interesting paper by P. Vermes [Amer. J. Math. vol. 71 (1949) pp. 541-562] appeared which overlaps the results of the present paper. In addition R. P. Agnew [Math. Rev. vol. 11, p. 242] in a review of a paper on these methods by W. König-Meyer [Math. Zeit. vol. 52 (1949) pp. 257-304] points out that the first systematic study of these methods is in the thesis of R. Wais [Das Taylorsche Summierungsverfahren, Tubingen, 1935].

\section{BiBLIOGRAPHY}

1. R. P. Agnew, Comparison of products of methods of summability, Trans. Amer. Math. Soc. vol. 43 (1938) pp. 327-343.

2. O. Toeplitz, Über allgemeine lineare Mittelbildungen, Prace MatematycznoFizyczne vol. 22 (1911) pp. 113-119.

LEHIGH UNIVERSITY 